\title{
UNIQUENESS THEOREMS FOR PERIODIC FUNCTIONS
}

\author{
N. J. FINE AND H. S. WILF ${ }^{1}$
}

1. Introduction. In this note we are concerned with theorems to the effect that two periodic functions which agree with each other on a "sufficiently large" set agree identically. Our criteria for "largeness" are best possible in all cases considered, namely the discrete case, the continuous case with commensurable periods and the continuous case with incommensurable periods. The results are given by the following three theorems.

TheOREM 1. Let $\left\{f_{n}\right\}_{0}^{\infty},\left\{g_{n}\right\}_{0}^{\infty}$ be two periodic sequences of periods $h$, $k$, respectively. If $f_{n}=g_{n}$ for $h+k-(h, k)$ consecutive integers $n$, then $f_{n}=g_{n}$ for all $n$. The result would be false if $h+k-(h, k)$ were replaced by anything smaller.

THEOREM 2. Let $f(x), g(x)$ be continuous periodic functions of periods $\alpha, \beta$, respectively, where $\alpha / \beta=p / q,(p, q)=1$, is rational. If $f(x)=g(x)$ on an interval of length $\alpha+\beta-\beta q^{-1}$, then $f(x) \equiv g(x)$. The result would be false if $\alpha+\beta-q^{-1} \beta$ were replaced by anything smaller.

TheOREM 3. Let $f(x), g(x)$ be continuous periodic functions of periods $\alpha, \beta$ respectively, where $\alpha / \beta$ is irrational. If $f(x)=g(x)$ on an interval of length $\alpha+\beta$, then $f(x) \equiv g(x)$. The result would be false if $\alpha+\beta$ were replaced by anything smaller.

2. Discrete case. The method we shall use for Theorems 2 and 3 also can be used to prove Theorem 1 . In this case, however, we give two different proofs, the first of which uses an argument which was pointed out by E. G. Straus and is perhaps the most direct. If $f_{n}=g_{n}$ $(n=0,1, \cdots, h+k-(h, k)-1)$ then

$$
\begin{aligned}
& F(x)=\sum_{0}^{\infty} f_{n} x^{n}=\left(1-x^{h}\right)^{-1} P(x), \\
& G(x)=\sum_{0}^{\infty} g_{n} x^{n}=\left(1-x^{k}\right)^{-1} Q(x),
\end{aligned}
$$

where $P(x), Q(x)$ are polynomials of degrees $\leqq h-1, k-1$, respectively. Then

Received by the editors August 7, 1963.

1 The work of the second author was supported in part by the National Science Foundation. 


$$
H(x)=F(x)-G(x)=\left(1-x^{(h, k)}\right)\left(1-x^{h}\right)^{-1}\left(1-x^{k}\right)^{-1} R(x),
$$

where $R(x)$ is a polynomial of degree $\leqq h+k-(h, k)-1$. Since the first $h+k-(h, k)$ coefficients of $H$ are $0, R(x) \equiv 0$ and $F(x) \equiv G(x)$, which was to be shown.

For another proof note that the most general periodic sequences of periods $h$ and $k$ are

$$
\begin{aligned}
& f_{n}=\sum_{\omega^{n=1}} c_{\omega} \omega^{n}, \\
& g_{n}=\sum_{\xi=1} d_{\xi} \xi^{n},
\end{aligned}
$$

the $c$ 's and $d$ 's being arbitrary. Now exactly $(h, k)$ of the w's are also $\xi$ 's and so the equations

$$
0=f_{n}-g_{n}=\sum c_{\omega} \omega^{n}-\sum d_{\xi} \xi^{n} \quad(n=1,2, \cdots, N)
$$

are $N$ equations in $h+k-(h, k)$ unknowns, namely, $c_{\omega}, d_{\xi}(\xi \neq \omega)$, $c_{\omega}-d_{\xi}(\omega=\xi)$. Hence if $N=h+k-(h, k)$, these equations have only the trivial solution since the coefficient matrix is a Vandermonde matrix formed from the distinct $\omega$ 's and $\xi$ 's and is therefore nonsingular.

This last argument also shows that the result is best possible since we will have a nontrivial solution for $N<h+k-(h, k)$. We can, however, be more constructive about this point by explicitly displaying a function $f(x)(x=0,1, \cdots)$ with the following properties:

(a) $f(x+k)=f(x)$ (all $x)$,

(b) $f(x+h)=f(x)(x=0,1, \cdots, k-3)$,

where $k, h$ are integers, $h<k,(h, k)=1$.

To do this define the function $\lambda$ of period $k$ by

$$
\lambda(x)= \begin{cases}1, & 0 \leqq x \leqq k-3, \\ 0, & k-2 \leqq x \leqq k-1\end{cases}
$$

and note that

$$
\lambda(x) \lambda(x-h) \lambda(x-2 h) \cdots \lambda(x-N h)=0
$$

for large enough $N$. Now let $\phi(x)$ be an arbitrary function satisfying

$(\alpha) \phi(x)=\phi(x+k)($ all $x)$,

( $\beta) \phi(x)=0,0 \leqq x \leqq k-3$.

Then the function

$$
\begin{aligned}
f(x)= & \phi(x)+\lambda(x) \phi(x+h)+\lambda(x) \lambda(x+h) \phi(x+2 h) \\
& +\cdots+\lambda(x) \cdots \lambda(x+(N-1) h) \phi(x+N h)
\end{aligned}
$$


has the required properties. If we define $g$ by

$$
g(x)=f(x) \quad(0 \leqq x \leqq h+k-3)
$$

and by periodicity of period $h$ for other $x$ then we have functions which show that our theorem is best possible for $(h, k)=1$. If $(h, k)$ $=d>1$ we first construct $f^{*}(x), g^{*}(x)$ as above for periods $h d^{-1}, k d^{-1}$. Each of the first $(h+k) / d-2$ values of $f^{*}(x), g^{*}(x)$ is then replaced by a block of length $d$ of the same value. The next value of $f^{*}, g^{*}$ is also replaced by a block of length $d$ whose first $d-1$ entries agree for $f$ and $g$ and whose last entry is different. These functions are then extended by periodicity and show that the theorem is best possible in this case also.

3. Continuous case, commensurable periods. Let $f(x), g(x)$ be continuous functions which are periodic, of periods $1, h=p / q<1$, $(p, q)=1$, respectively. For each $x \in\left[0, q^{-1}\right]$ define

$$
\begin{aligned}
& a_{n}=f\left(x+\frac{n}{q}\right), \\
& b_{n}=g\left(x+\frac{n}{q}\right),
\end{aligned}
$$

for all integers $n$. Then $a_{n}, b_{n}$ are sequences of periods $q, p$, respectively. By Theorem 1, if

$$
a_{n}=b_{n} \quad(n=0,1, \cdots, p+q-2),
$$

then $a_{n}=b_{n}$ for all $n$. Hence if $f(x)=g(x)$ for all $x \in[0,(p+q-1) / q)$ $=\left[0,1+h-q^{-1}\right)$ then $f=g$. This proves the first part of Theorem 2 . To see that the constant is best possible it is enough to construct a continuous function $f(x)$ such that

(a) $f(x+1)=f(x)$ (all $x)$,

(b) $f(x+h)=f(x)\left(0 \leqq x \leqq 1-q^{-1}-\epsilon\right)$.

\section{Define}

$$
\lambda(x)= \begin{cases}1, & 0 \leqq x \leqq 1-q^{-1}-\epsilon, \\ 0, & 1-q^{-1}-\epsilon<x<1, \\ \lambda(x+1), & \text { all } x .\end{cases}
$$

Then we need

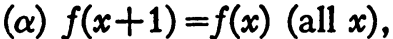

(B) $\lambda(x) f(x)=\lambda(x) f(x+h)$ (all $x)$.

Let $\phi(x)$ be a continuous function satisfying 


$$
\phi(x+1)= \begin{cases}\phi(x), & \text { all } x, \\ 0, & 0 \leqq x \leqq 1-q^{-1}-\epsilon .\end{cases}
$$

We claim that, with a suitable choice of $\phi$,

$$
f(x)=\phi(x)+\lambda(x) \phi(x+h)+\lambda(x) \lambda(x+h) \phi(x+2 h)+\cdots
$$

has the required properties. In fact if, for some $n$,

$$
H(x)=\lambda(x) \lambda(x+h) \cdots \lambda(x+n h)=0 \quad(\text { all } x),
$$

then the series is finite.

Let $I=\left(1-q^{-1}-\epsilon, 1\right)$. Then $H(x)=0$ for $x$ in $I \cup(I-h) \cup(I-2 h)$ $\cup \cdots \cup(I-n h)(\bmod 1)$. The right-hand end points of these intervals are $1,1-h, \cdots, 1-n h(\bmod 1)$. These form a periodic sequence of period $q$, and run through the points $-j p q^{-1}(\bmod 1)$. Since $(p, q)$ $=1$, given $r$ we can solve

$$
\begin{aligned}
-j p & \equiv r(\bmod q), \\
-j \frac{p}{q} & \equiv \frac{r}{q}(\bmod 1) .
\end{aligned}
$$

Thus the entire unit interval is covered.

To see that $f$ satisfies $(\alpha),(\beta)$, note that each term has period 1 and, further,

$$
\begin{aligned}
\lambda(x) f(x+h) & =\lambda(x) \phi(x+h)+\lambda(x) \lambda(x+h) \phi(x+2 h)+\cdots \\
& =\lambda(x)(f(x)-\phi(x)) \\
& =\lambda(x) f(x) .
\end{aligned}
$$

For continuity we add the condition that $\phi(x)=0$ at the finite set of points at which $\lambda(x), \lambda(x-h), \cdots$ are discontinuous.

Having $f(x)$ we put

$$
g(x)= \begin{cases}f(x), & 0 \leqq x \leqq h, \\ g(x+h), & \text { all } x .\end{cases}
$$

Then for $0 \leqq x \leqq 1+h-q^{-1}-\epsilon$ there exists an $n$ such that

$$
0 \leqq x-n h<h \text {. }
$$

Then

$$
g(x)=g(x-n h)=f(x-n h)=\cdots=f(x-h)=f(x) .
$$

4. Continuous case, incommensurable periods. If $f(x), g(x)$ are continuous functions of periods $1, \theta(\theta$ irrational, $\theta<1)$ and if $f(x)$ 
$=g(x)$ for $0 \leqq x \leqq 1+\theta$, then we claim that $f(x), g(x)$ are both (the same) constant. Let $0 \leqq x<\theta$. We show that

$$
f(x)=f(\{x+m \theta\}) \quad(m=0,1, \cdots) .
$$

This is obvious for $m=0$. If proved for $m=k$, then

$$
\begin{aligned}
f(x) & =f(\{x+k \theta\}) \\
& =g(\{x+k \theta\}) \\
& =g(\theta+\{x+k \theta\}) \\
& =f(\theta+\{x+k \theta\}) \\
& =f(\theta+x+k \theta) \\
& =f(\{x+(k+1) \theta\}) .
\end{aligned}
$$

Since $\{x+m \theta\}$ is dense in $[0,1]$ and $f$ is continuous, $f(x)$ is constant on $[0,1]$ and therefore identically. Consequently $g(x)$ is identically this same constant.

To show that the theorem is best possible we construct, as before, a continuous $f(x)$ satisfying

(a) $f(x+1)=f(x)$ (all $x)$,

(b) $f(x+\theta)=f(x)(0 \leqq x \leqq 1-\epsilon)$,

where $0<\epsilon<1$.

We put

$$
\lambda(x)= \begin{cases}1, & 0 \leqq x \leqq 1-\epsilon, \\ 0, & 1-\epsilon<x<1, \\ \lambda(x+1), & \text { all } x .\end{cases}
$$

Let $I$ be $[1-\epsilon, 1]$. The sets $I+n \theta(\bmod 1)$ cover $[0,1]$. Hence there is an $N$ such that $I+n \theta, \bmod 1, n=0, \cdots, N$, cover $[0,1]$. Thus

$$
\lambda(x) \lambda(x-\theta) \lambda(x-2 \theta) \cdots \lambda(x-N \theta)=0 .
$$

Let $E$ be the set of points of discontinuity of all the functions $\lambda(x)$, $\lambda(x-\theta), \cdots, \lambda(x-N \theta) . E$ is finite. Now let $\phi(x)$ be any function satisfying

$$
\phi(x)= \begin{cases}\phi(x+1), & \text { all } x, \\ 0, & 0 \leqq x \leqq 1-\epsilon, \\ 0, & x \in E .\end{cases}
$$

Then 


$$
\begin{aligned}
f(x)= & \phi(x)+\lambda(x) \phi(x+\theta)+\cdots \\
& +\lambda(x) \lambda(x+\theta) \cdots \lambda(x+(N-1) \theta) \phi(x+N \theta)
\end{aligned}
$$

is continuous, and it is easy to check that it is a solution of our problem. Finally, $g$ is defined as the function of period $\theta$ which agrees with $f$ on $[0, \theta]$. Here, as in the other two cases, $\phi$ can be chosen so that $f \neq g$.

We remark that an alternate approach to the last construction leads to an interesting class of problems in harmonic analysis. Let $f(x)$ be the required function, and define $\alpha(x)=f(x+\theta)-f(x)$. Then $\alpha$ is continuous, with period 1 , and has its support in $(1-\epsilon, 1) \bmod$ 1. If

$$
f \sim \sum c_{n} e^{2 \pi i n x}
$$

and

$$
\alpha \sim \sum a_{n} e^{2 \pi i n x}
$$

then

$$
c_{n}=\frac{a_{n}}{e^{2 \pi i n \theta}-1} .
$$

Thus it is not difficult to see that our problem is equivalent to the following.

Find a nonzero continuous function $\alpha(x)$ such that:

$$
\begin{aligned}
\alpha(x+1) & =\alpha(x) \quad \text { (all } x), \\
\text { Supp } \alpha & \subset(1-\epsilon, 1) \bmod 1, \\
c_{n} & =\left(e^{2 \pi i n}-1\right)^{-1} \int_{1-\bullet}^{1} \alpha(x) e^{-2 \pi i n x} d x
\end{aligned}
$$

are the Fourier coefficients of a continuous function $f$. It is not obvious, a priori, that a function $\alpha$ with support restricted by (2) can have its Fourier coefficients small enough for those $n$ such that $\{n \theta\}$ is close to 0 mod 1. "Small enough" means condition (3).

Our proof, of course, shows that such a function does exist.

University of Pennsylvania 